\title{
Diagnosis versus Detection of Caries
}

\author{
B. Nyvad \\ School of Dentistry, Faculty of Health Sciences, University of Aarhus, Aarhus, Denmark
}

\section{Key Words}

Dental caries, detection. Dental caries, diagnosis . Diagnostic threshold · 'Gold standard' · Lesion activity · Randomized controlled trial · Reliability · Treatment decision · Validity

\begin{abstract}
Caries diagnosis is the art or act of identifying a disease from its signs and symptoms. This is distinct from the detection of the signs and symptoms themselves. The diagnosis forms the basis for making informed treatment decisions. Hence, if there is no diagnostic step expressed in terms of the probability of present and future occurrence of disease, practitioners may resort to treatments guided by previous experiences with similar clinical manifestations. This paper reviews various methodological aspects of caries diagnostic testing. It is concluded that rather than continuing to search for the truth of the diagnosis, it may be more informative to consider the consequences of the diagnosis. This view is supported by results from caries-preventive trials in which the activity of carious lesions has been monitored longitudinally over years.
\end{abstract}

Copyright @ 2004 S. Karger AG, Base

ORCA was among the first scientific fora to focus on the challenges of diagnosing caries in populations with low rates of lesion progression. In the concluding remarks of the Symposium of the ORCA Caries Diagnosis Working Group in Helsinki in 1992 it was stated: 'The development of methods for determining whether a carious lesion is stable or progressing is a priority in caries research and a mechanism in terms of estimated progression will be invaluable. Detection of small carious lesions is only part of the problem, the appropriate method for therapeutic and preventive care that can be applied to the lesion is the aspect facing the clinician daily' [Pine and ten Bosch, 1996]. Regrettably, in spite of these promising visions, most research on caries diagnosis since then has concentrated on the development and testing of new methods for the detection of small lesions. There has been almost no focus on whether the use of such new methods may facilitate treatment decisions or lead to better health outcomes for the patients.

The purpose of this paper is first to revisit some issues pertaining to the evaluation of caries diagnostic tests. Secondly, to highlight some new developments within the field of clinical caries diagnosis that may have important implications for caries diagnostic decision making and the delivery of non-operative preventive care.

\section{To Diagnose or to Detect?}

The art of diagnosis rests on the assumption that diseases can be distinguished from their signs and symptoms. Diagnostic reasoning is an extremely complex process that involves elements of simple pattern recognition,

\begin{tabular}{ll}
\hline KARGER & ( 2004 S. Karger AG, Basel \\
Fax +4161306 12 34 & 0008-6568/04/0383-0192\$21.00/0 \\
$\begin{array}{l}\text { E-Mail karger@karger.ch } \\
\text { www.karger.com }\end{array}$ & $\begin{array}{l}\text { Accessible online at: } \\
\text { www.karger.com/cre }\end{array}$
\end{tabular}

Bente Nyvad

School of Dentistry, Faculty of Health Sciences

University of Aarhus, Vennelyst Boulevard

DK-8000 Aarhus C (Denmark)

Tel. +45 89424074, Fax +45 86202202, E-Mail nyvad@odont.au.dk 


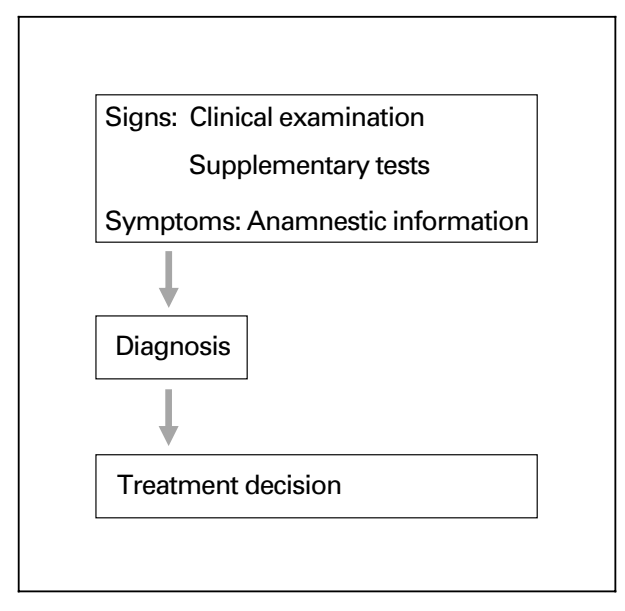

Fig. 1. The classical diagnostic decision process.

probabilistic considerations and hypothetico-deductive thinking [Wulff and Gøtzsche, 2000]. Diagnostic decision making is a balancing act. The clinician must not overlook diseases in need of treatment, and, at the same time, he must not make a diagnosis when it is not warranted. The choice of diagnosis is further complicated by the fact that the clinician must take into account the consequences for the patient. The inherent complexity of the diagnostic process explains why nobody has ever been able to unveil how clinicians think when they examine their patients and seek the right diagnosis. It also explains why diagnostic skills cannot be learnt from textbooks alone, but require clinical training.

Due to the deductive nature of the diagnostic process, the term diagnosis should not be used synonymously with the term detection. To diagnose is 'the art or act of identifying a disease from its signs and symptoms' [MerriamWebster, 2003]. This is distinct from the detection of the signs and symptoms themselves. During the diagnostic process the clinician attempts to assign a label to a set of signs and symptoms brought together from various sources (e.g. interview, clinical examination and supplementary tests). This information is used to assess the probability that the patient has a certain condition. In medicine the diagnosis is a pivotal step for making treatment decisions. Therefore, the diagnostic step has sometimes been referred to as 'a mental resting place on the way to intervention' [Baelum and Fejerskov, 2003]. Figure 1 illustrates the classical diagnostic decision process as outlined above.

It has been proposed that the diagnostic problem in caries differs from that of other medical diseases. Hence, because there are virtually no symptoms of caries (at least in the early stages of the disease process!) it has been claimed that there is also no diagnostic 'step' and consequently no diagnosis in caries [Bader and Shugars, 1996]. Caries examination becomes primarily a question of detection (caries yes or no). This concept may have led to the unfortunate situation that dentists have merged their diagnostic activities with treatment planning decisions [Beck, 1995]. Moreover, it may explain why we have never had a true evidenced-based approach to non-operative preventive caries treatment. Thus, if there is no diagnosis, expressed in terms of the probability of present or future occurrence of caries, practitioners may easily resort to a treatment pattern where previous experiences with similar clinical manifestations will guide the choice of treatment.

\section{How to Evaluate a Caries Diagnostic Test?}

In view of the above considerations it is not sufficient to have a good diagnostic test; the test must also work well [Bader and Shugars, 1996]! Traditionally, the performance of a diagnostic test is evaluated in terms of its validity (the degree to which a measurement measures what it purports to measure with reference to an independent 'gold standard') and reliability (the degree to which the results obtained by a measurement procedure can be replicated) [Last, 1995]. However, it is also important to assess the consequences for the patient in response to the diagnosis and/or treatment provided. Last but not least, the evaluation should include considerations about the potential negative aspects for the patient as well as considerations about the cost-effectiveness of using the test. Only when all the above issues have been adequately addressed, should a new diagnostic test or tool be implemented for routine use in clinical practice [Abramson, 1990].

\section{The 'Gold Standard'}

Validity may be appraised in different ways although in caries criterion validity has been the most commonly applied. Assessment of criterion validity requires the existence of external criteria - so-called 'gold standards' - for the phenomenon. So far most evaluations of caries diagnostic criteria have focused on depth of lesion penetration as assessed either histologically, clinically or radiographically [Hintze and Wenzel, 2003]. There is no strict rule as 
to what the 'gold standard' should be; it is a matter of choice [ten Bosch and Angmar-Månsson, 2000]. The investigator should always choose a 'gold standard' that represents the highest level of truthfulness for the phenomenon under study. Depth of lesion penetration may not necessarily be the most appropriate 'gold standard' for caries. Furthermore, for practical or ethical reasons it may not always be possible to use the most exact 'gold standard'. Researchers and clinicians should be aware of this when evaluating the performance of caries diagnostic tests. A new diagnostic method that compares favourably with an accepted 'gold standard' can be no better than the 'gold standard' being used. Likewise, if a new method fails to compare favourably with a 'gold standard', the test may not necessarily be useless. It may in fact be superior to the 'gold standard' used [Beck, 1995].

In the absence of an external 'gold standard', validity can sometimes be appraised by seeing whether a followup study shows an association between the measurement and a subsequent event (e.g. cavity formation) that is believed to be an outcome of what was measured (predictive validity). Another way of appraising validity is to see whether there are associations with other variables that there is reason to believe should be linked with the characteristic under study (construct validity) [Abramson, 1990]. Recently, the latter methods were successfully used to determine the validity of caries activity assessments in a clinical trial of the preventive effect of fluoride toothpaste [Nyvad et al., 2003].

\section{Qualitative versus Quantitative Methods}

When summarizing the data from studies that have assessed criterion validity of current clinical caries diagnostic methods (e.g. conventional clinical examination and radiography) most of the studies have shown a rather poor validity with low sensitivity and moderate specificity [for reviews, see Pine and ten Bosch, 1996; Ismail, 1997; Bader et al., 2002]. This implies that caries diagnosis, as normally performed in daily clinical practice, is an inexact procedure that results in both over- and underdiagnosis. In reality, the diagnostic performance may be much poorer than assumed because the majority of the validation studies have been performed under laboratory conditions, excluding the difficulties introduced by the presence of plaque and saliva.

The rather poor diagnostic performance of the conventional caries diagnostic methods has prompted some researchers to search for quantitative detection methods, such as electrical conductance measurements, light scattering methods, laser fluorescence methods. There were at least three motives for this development: (1) that quantitative methods may detect lesions at an earlier stage than conventional methods; (2) that quantitative measurements are more reliable than qualitative measurements, and (3) that quantitative assessments may provide the means for non-destructive monitoring of the course of disease [ten Bosch and Angmar-Månsson, 2000]. Indeed, some quantitative detection methods have shown a high degree of reliability [Lussi et al., 1999; Tranaeus et al., 2002]. Likewise, it has been demonstrated that quantitative light-induced fluorescence methods may be suitable for the longitudinal quantification of non-cavitated carious lesions on smooth tooth surfaces, especially for scientific purposes [Angmar-Månsson et al., 1996; Tranaeus et al., 2001]. However, laser fluorescence suffers from a poor correlation with mineral loss [Shi et al., 2001]. Furthermore, the cut-off points used to classify the numerical output of the latter devices into sound, enamel and dentin caries have not been finally determined in vivo [Lussi et al., 2001]. Therefore, until such methods have been properly tested and have proved superior to conventional caries diagnostic methods, such as clinical examination [Attrill and Ashley, 2001], they should not be recommended for routine clinical use.

\section{How Early Should a Lesion Be Detected?}

Along with the development of quantitative methods for caries lesion detection there has been a parallel effort to detect lesions as early as possible, even prior to the non-cavitated stage of lesion formation [Stookey, 2000; Pitts, 2001]. This development has been driven by the belief that early identification provides better estimates of disease and improves the possibility for successful preventive intervention. However, from a clinical point of view this can be questioned. A low diagnostic threshold not only leads to detection of more small lesions but also to more false positive diagnoses. Furthermore, because a significant number of the early lesions are likely to arrest or regress without professional intervention [Backer Dirks, 1966; Baelum et al., 2003], such a strategy may not be cost-effective. Setting the diagnostic threshold is therefore a question of balance. At present, for clinical purposes it would seem essential to diagnose lesions at a non-cavitated stage but not at such an early stage that they are invisible to the naked eye. This position is supported by the observation that non-cavitated carious 
Fig. 2. The relative risk $(R R)$ that a fluorideexposed surface versus a control surface undergoes lesion transition, as observed in a 3year caries-preventive trial of daily supervised brushing with fluoride toothpaste in young teenagers. $R R<1$ indicates inhibition of lesion progression; RR $>1$ indicates promotion of lesion regression. Data modified after Nyvad et al. [2003].

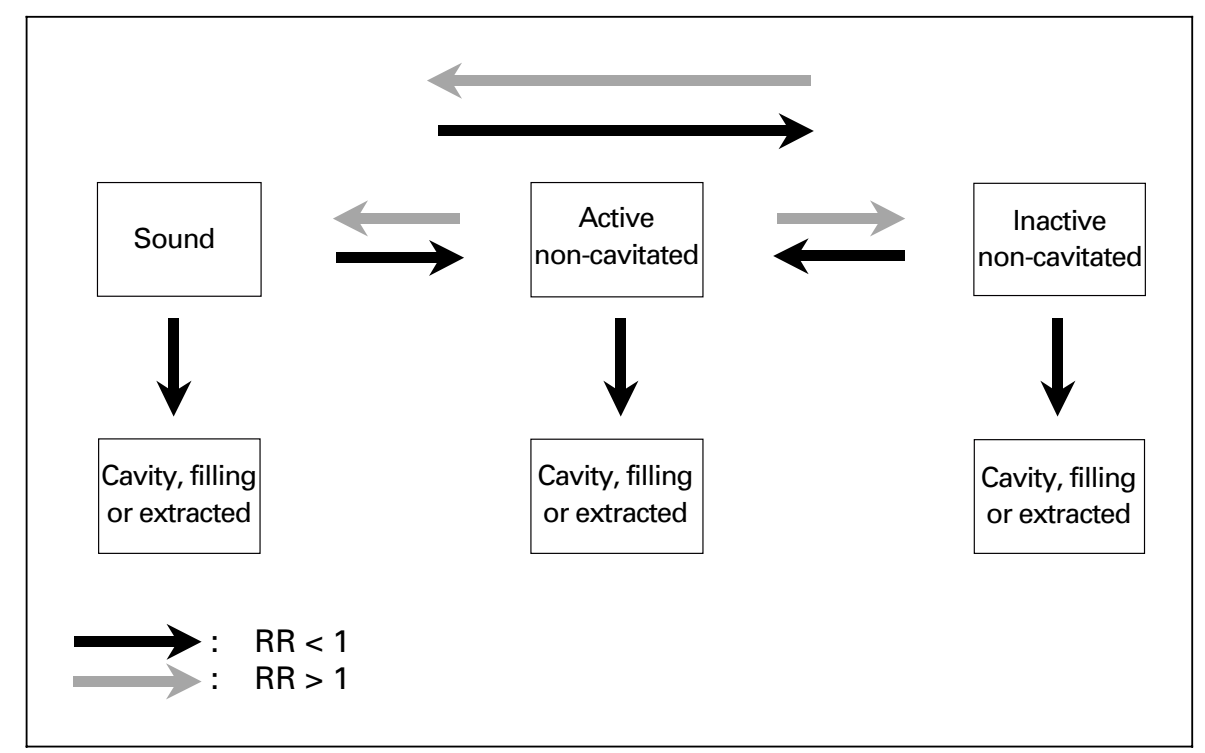

lesions can be controlled sufficiently by non-operative preventive interventions such as topical fluorides [Marinho et al., 2003].

\section{Recent Developments in Clinical Caries Diagnosis}

Because of low rates of lesion progression in many Western countries there has over the past decade been an interest in developing clinical diagnostic criteria for assessing the activity state of non-cavitated carious lesions. The theoretical foundation of one such system [Nyvad et al., 1999] is the assumption that lesion activity, defined as net progression or net regression [Fejerskov and Manji, 1990], will be reflected in the surface features of the lesion: matt, 'chalky' and rough enamel lesions being 'active', and shiny, smooth enamel lesions being 'inactive' or 'arrested' [for review, see Thylstrup et al., 1994]. By definition, these categories refer to the activity state of the lesion as inferred at the time of examination. Such distinctions have no bearing on what may occur to the lesion over time. Hence, if an 'active' lesion is subjected to efficacious preventive intervention, such as oral hygiene or topical fluorides, the activity state of the lesion is likely to change [Baelum et al., 2003]. Only if the local environmental conditions of a lesion remain unchanged can the activity state be expected to stay the same.

Clinical studies using this new diagnostic method in contemporary North-European child populations have shown that caries activity assessments may be both reliable and valid, regardless of the diagnostic threshold chosen. In well-trained examiners the kappa values ranged between 0.74 and 0.85 for intra-examiner agreement and between 0.78 and 0.80 for inter-examiner agreement [ $\mathrm{Ny}-$ vad et al., 1999]. The pattern of distribution of the diagnoses showed that the diagnostic criteria were effective in separating 'active' from 'inactive' lesions. However, as with other studies assessing non-cavitated carious lesions [Ismail et al., 1992], it was often difficult to differentiate between a diseased surface and a sound surface, possibly because of insufficient plaque removal prior to the examination.

Due to the lack of an independent 'gold standard' for caries activity, construct validity of the criteria was evaluated by means of the ability of the criteria to reflect known effects of fluoride on caries [Nyvad et al., 2003]. When reanalysing the results from a 3-year trial of daily supervised brushing with fluoride toothpaste [Machiulskiene et al., 2002] it was found that the relative risks for lesion transitions in the experimental group versus the control group imitated the anticipated effect of fluoride on caries lesion dynamics [ten Cate and Featherstone, 1996]; hence, at all stages of lesion formation brushing with fluoride toothpaste inhibited the development or progression of caries and at the same time enhanced arrest or regression of caries (fig. 2). These effects were most pronounced for 'active' non-cavitated lesions supporting the notion that fluoride exerts its predominant effect on the active caries process [Fejerskov et al., 1981]. It was concluded, 


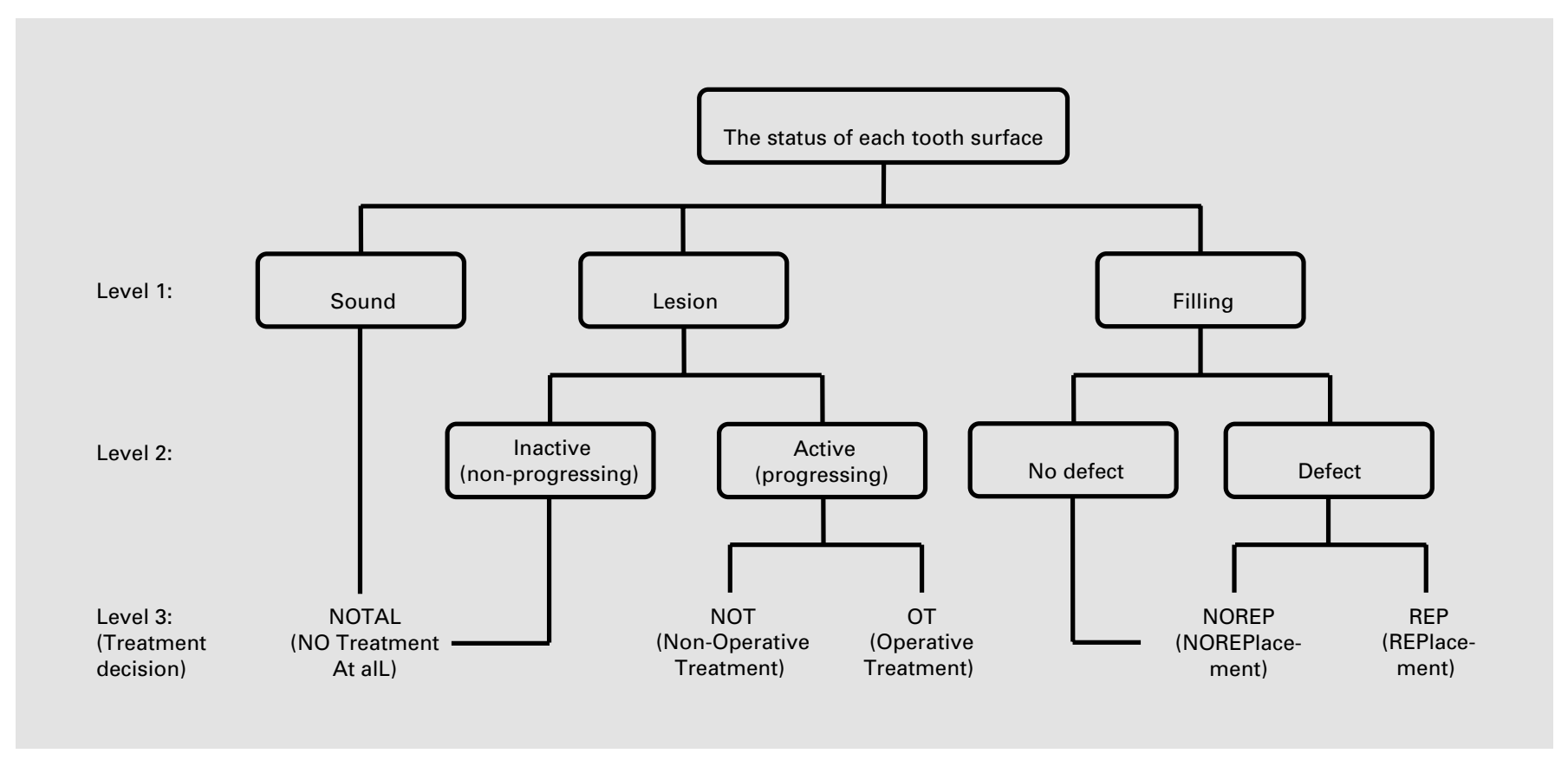

Fig. 3. Decision-making tree for dental caries according to Nyvad and Fejerskov [1997]. Note that the flow chart does not take into account factors operating at the level of the individual.

therefore, that the diagnostic criteria had construct validity for the assessment of caries lesion activity.

A further finding of the study was that caries activity assessments had predictive validity [Nyvad et al., 2003]. Thus, 'active' non-cavitated lesions had a considerably greater risk of progressing to a cavity than 'inactive' noncavitated lesions, an effect that was more pronounced in subjects not regularly exposed to fluoride. The latter observation implies that caries activity assessments may be used to advise the subsequent course of treatment. In fact, we have previously proposed a decision-making tree for dental caries that has included activity assessment as a key factor in the decision process (fig. 3) [Nyvad and Fejerskov, 1997]. According to this model the first level of the decision process is to determine the state of the tooth surface, whether sound, having a lesion or being filled. Suppose it has been decided that there is a lesion, one may proceed to level 2, which is to assess the activity state of the lesion: 'inactive' (non-progressing) or 'active' (progressing). The third step is to generate a proper treatment decision. 'Inactive' lesions, because of their slowly or nonprogressing nature, do not need professional treatment. 'Active' lesions, on the other hand, because of their progressive nature, demand professional treatment. The professional treatment of 'active' lesions will depend on the accessibility to plaque removal. If plaque control is likely to be insufficient, such as in cavitated lesions, the lesion should be filled. However, for non-cavitated 'active' lesions, non-operative preventive interventions are to be preferred. This example illustrates how activity assessment could serve as an important diagnostic means for making evidence-based treatment decisions.

\section{Concluding Remarks}

When dealing with diagnostic questions one should bear in mind that the diagnosis is not a goal by itself. The ultimate goal of making a diagnosis is always to select the best possible treatment. Except for the above systematic approach, researchers have shown very little interest in the long-term outcomes of caries diagnostic procedures. This is surprising as for both the clinician and the patient the outcome in terms of the appropriateness of the treatment provided in response to a given diagnosis is of principal interest [Bader et al., 2002; Baelum and Fejerskov, 2003]. Future research on caries diagnosis should be much more concerned about evaluating the long-term consequences of diagnostic methods (e.g. in response to refined diagnostic methods or different modes of lesion 


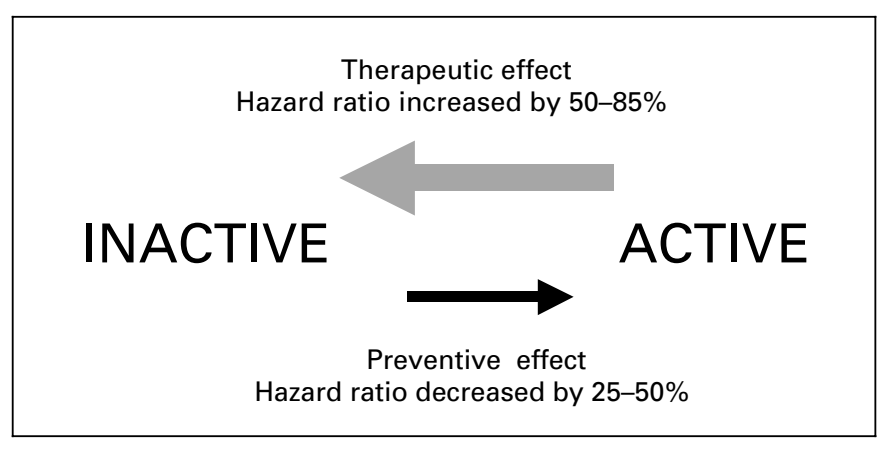

Fig. 4. The effect of fluoride on hazard ratios for caries transitions from inactive to active and active to inactive state, respectively, as observed in a 3-year caries-preventive trial of daily supervised brushing with fluoride toothpaste in young teenagers. Percentages refer to different types of tooth surfaces. Data modified after Baelum et al. [2003].

classification) than by studying the immediate outcomes in terms of accuracy and reliability. This is not to say that the latter issues are not important. However, if we appreciate that the performance of most caries diagnostic tools is poor, it may be more pertinent to consider the conse- quences of the diagnosis than to dwell on the truth of the diagnosis [Wulff, 1979].

The views presented here imply that in addition to assessing validity and reliability, the performance of caries diagnostic methods should also be evaluated in randomized controlled trials. Such a methodology may, in fact, have additional benefits. We were recently fascinated to learn how survival time analysis of lesion transitions in a caries-preventive trial [Machiulskiene et al., 2002] provided novel information about the preventive and therapeutic actions of fluoride. Thus, when lesions were dichotomized into 'inactive' and 'active' stages the hazard ratios for transitions from 'inactive' to 'active' were decreased by $25-50 \%$ in the fluoride group compared to the control group while the corresponding ratios for transitions from 'active' to 'inactive' were increased by $50-85 \%$, depending on the type of surface examined (fig. 4) [Baelum et al., 2003]. Collectively, these observations suggest that the therapeutic effect of fluoride may by far exceed the preventive effect, an observation that is in concert with the marked retardation of caries progression beyond the stage of enamel caries in individuals living in water-fluoridated areas [Groeneveld, 1985].

\section{References}

Abramson JH: Survey Methods in Community Medicine: Epidemiological Studies, Programme Evaluation, Clinical Trials, ed 4. New York, Churchill Livingstone, 1990, pp 47-56 151-164.

Angmar-Månsson $\mathrm{B}$, Al-Khateeb $\mathrm{S}$, Tranaeus S: Monitoring the caries process: Optical methods for clinical diagnosis and quantification of enamel caries. Eur J Oral Sci 1996;104:480 485.

Attrill DC, Ashley PF: Occlusal caries detection in primary teeth: A comparison of DIAGNOdent with conventional methods. Br Dent J 2001; 190:436-443.

Backer Dirks O: Posteruptive changes in dental enamel. J Dent Res 1966;45:503-511.

Bader JD, Shugars DA: Issues in the adoption of new methods of caries diagnosis; in Stookey GK (eds): Early Detection of Dental Caries. Indianapolis, Indiana University School of Dentistry, 1996, pp 11-26.

Bader JD, Shugars DA, Bonito AJ: A systematic review of the performance of methods for identifying carious lesions. J Public Health Dent 2002;62:201-213.

Baelum V, Fejerskov O: Caries diagnosis: 'A mental resting place on the way to intervention'?; in Fejerskov O, Kidd E (eds): Dental Caries: The Disease and Its Clinical Management. Oxford, Blackwell Munksgaard, 2003, pp 101-110.
Baelum V, Machiulskiene V, Nyvad B, Richards A, Væth M: Application of survival analysis to carious lesion transition in intervention trials. Community Dent Oral Epidemiol 2003;31: 252-260.

Beck JD: Issues in assessment of diagnostic tests and risk for periodontal diseases. Periodontol 2000 1995; 7:100-108.

ten Bosch JJ, Angmar-Månsson B: Characterization and validation of diagnostic methods; in Faller RV (ed): Assessment of Oral Health. Monogr Oral Sci Basel, Karger 2000, vol 17, pp 174-189.

ten Cate JM, Featherstone JDB: Physicochemical aspects of fluoride-enamel interactions; in Fejerskov O, Ekstrand J, Burt BA (eds): Fluoride in Dentistry. Copenhagen, Munksgaard, 1996, pp 252-272.

Fejerskov O, Manji F: Risk assessment in dental caries; in Bader JD (ed): Risk Assessment in Dentistry. Chapel Hill, University of North Carolina Dental Ecology, 1990, pp 215-217.

Fejerskov O, Thylstrup A, Larsen MJ: Rational use of fluorides in caries prevention: A concept based on possible cariostatic mechanisms. Acta Odontol Scand 1981;39:241-249.

Groeneveld A: Longitudinal study of prevalence of enamel lesions in a fluoridated and non-fluoridated area. Community Dent Oral Epidemiol 1985;13:159-163.
Hintze H, Wenzel A: Diagnostic outcome methods frequently used for caries validation. Caries Res 2003;37:115-124.

Ismail AI: Clinical diagnosis of precavitated carious lesions. Community Dent Oral Epidemiol 1997;25:13-23.

Ismail AI, Brodeur J-M, Gagnon P, Payette M, Picard D, Hamalian T, Oliver M, Eastwood BJ : Prevalence of non-cavitated and cavitated carious lesions in a random sample of 7-9-yearold schoolchildren in Montreal, Quebec. Community Dent Oral Epidemiol 1992;20:250255.

Last JM: A Dictionary of Epidemiology. ed 3. Oxford, Oxford University Press, 1995.

Lussi A, Imwinkelried S, Pitts N, Longbottom C, Reich E: Performance and reproducibility of a laser fluorescence system for detection of occlusal caries in vitro. Caries Res 1999;33:261266.

Lussi A, Megert B, Longbottom C, Reich E, Francescut P: Clinical performance of a laser fluorescence device for detection of occlusal caries lesions. Eur J Oral Sci 2001;109:14-19.

Machiulskiene V, Richards A, Nyvad B, Baelum V: Prospective study of the effect of post-brushing rinsing behaviour on dental caries. Caries Res 2002;36:301-307. 
Marinho VC, Higgins JP, Sheiham A, Logan S: Fluoride toothpastes for preventing dental caries in children and adolescents. Cochrane Database Syst Rev 2003;1:CD002278.

Merriam-Webster 2003:www2.Merriam-webster. com/cgi-bin/mwmednlm.

Nyvad B, Fejerskov O: Assessing the stage of caries lesion activity on the basis of clinical and microbiological examination. Community Dent Oral Epidemiol 1997;25:69-75.

Nyvad B, Machiulskiene V, Baelum V: Reliability of a new caries diagnostic system differentiating between active and inactive caries lesions. Caries Res 1999;33:252-260.

Nyvad B, Machiulskiene V, Baelum V: Construct and predictive validity of clinical caries diagnostic criteria assessing lesion activity. J Dent Res 2003;82:117-122.
Pine CM, ten Bosch JJ: Dynamics of and diagnostic methods for detecting small carious lesions. Caries Res 1996;30:381-388.

Pitts NB: Clinical diagnosis of dental caries: A European perspective. J Dent Educ 2001;65: 973-980.

Shi XQ, Tranaeus S, Angmar-Månsson B: Comparison of QLF and DIAGNOdent for quantification of smooth surface caries. Caries Res 2001; 35:21-26.

Stookey GK: Practical applications of early caries detection methods; in Stookey GK (ed): Early Detection of Dental Caries. II. Indianapolis, Indiana University School of Dentistry, 2000, pp 357-363.

Thylstrup A, Bruun C, Holmen L: In vivo caries models - mechanisms for caries initiation and arrestment. Adv Dent Res 1994;8:144-157.
Tranaeus S, Al-Khateeb S, Bjorkman S, Twetman S, Angmar-Mansson B: Application of quantitative light-induced fluorescence to monitor incipient lesions in caries-active children: A comparative study of remineralization by fluoride varnish and professional cleaning. Eur J Oral Sci 2001;109:71-75.

Tranaeus S, Shi XQ, Lindgren LE, Trollsas K, Angmar-Mansson B: In vivo repeatability and reproducibility of the quantitative light-induced fluorescence method. Caries Res 2002;36:3-9.

Wulff HR: What is understood by a disease entity? J R Coll Physicians Lond 1979;13:219-220.

Wulff HR, Gøtzsche PC: Rational Diagnosis and Treatment: Evidence-Based Clinical Decision Making, ed 3. Oxford, Blackwell Science, 2000 . 